\title{
EXPERIMENTAL INVESTIGATION OF THE INFLUENCE OF MECHANICAL FORCED VIBRATIONS AND HEAT FLUX ON COEFFICIENT OF HEAT TRANSFER
}

\author{
Adil Bash ${ }^{\mathrm{a}, *}$, Aadel Alkumait ${ }^{\mathrm{a}}$ and Hamza Yaseen ${ }^{\mathrm{a}}$ \\ ${ }^{a}$ Mechanical engineering, engineering college, Tikrit university, Tikrit, Iraq (adelbash@tu.edu.iq)
}

\begin{abstract}
:
The aim of this paper to verify the influence of vertical forced vibration on the coefficient of heat transfer of the laminar internal flow in a spiral fluted tube. With adopted the water as a working fluid, and flowing Reynolds numbers at the entrance between 228 and 1923, the tube heated under constant heat flux levels ranging from $618-3775 \mathrm{~W} / \mathrm{m}^{2}$. The frequencies of vibration ranging from 13 to $30 \mathrm{~Hz}$, and the amplitudes of vibration from 0.001 to $0.002 \mathrm{~mm}$. The results appeared that the coefficient of heat transfer significantly affected by mechanical forced vibration in a flowing of the heated tube. When the vibration amplitude increases, the Nusselt number Significantly increases, with the maximum increases of $8.4 \%$ at the amplitude of vibration $0.0022 \mathrm{~mm}$ and the frequency $13 \mathrm{~Hz}$. Generally, the coefficient of heat transfer increases with increasing Reynolds number and heat flux. At last, by using the parameters of vibration amplitude, frequency, heat flux and Reynolds number, a new correlation has been derived depends on experimental data.
\end{abstract}

KEYWORDS: Frequency of Vibration, Amplitude, Non-Dimensional Coefficient of Heat Transfer, Reynolds Number.

\section{INTRODUCTION}

There are many vibrations and oscillation phenomena that happen in the industrial domain such pulse of flowing fluid and vibrations in equipment of mechanical, vibrations caused by sound, flutter of spacecraft, and vibrations due to ultrasound. A little of the influences of this oscillations cause fatigue stress due to vibration of constituent materials [1]. However, Some of These influences are useful, as mechanical vibrations are destabilizing in the processes of fluid flowing and heat transfer, thus increasing the amount of heat transferred. Neglecting of the effect of vibration on heat transfer in industrial equipment may lead to many constraints in dealing with applied cases [1]. The flow of tube is exceedingly used in different transfer systems of energy as food processing, reactions of chemical and pre-heating of materials. The process of heat transfer greatly affected by Thermal conductivity, and there is a simple heat transfer by convective between the various layers of the fluid. Achieving higher heat transfer is a great importance to the thermal process in the laminar flow region. The thermal performance improvement of the laminar internal flow is largely done by extension of surface as fins or surface roughness such as using spiral fluted tube. However, the focus on improving the surface lead to rising the losses of friction for the inner surface of tube [1].

The techniques of improving heat transfer depend mainly on reducing the thermal resistance that represents the impedance of the heat exchange process with or without increasing the area of the surface. The techniques of heat transfer improvement can generally be classified as passive and active techniques. The passive techniques include surface treatments, the surface alteration, extension of surfaces and roughening or use of an industrial substance or device to increase heat transfer rate. Active techniques contain vibration of surface, vibration of fluid, mechanical aids and electro-hydrodynamics where external energy is used mainly to modify the flow accompanied by an improvement in the rate of heat transfer. The influence of vibration on the process of heat transfer and internal flow has been studied by many researchers [2].

In experimental approaches, Bronfenbrener et.al. [3] studied the influence of vibration to enhance the rate of heat transfer from a circular tube surrounded by a pipe with rings distributed on the inner tube and with frequencies of vibration ranging from $10 \mathrm{~Hz}$ to $120 \mathrm{~Hz}$, and vibration amplitude ranging from $0.1 \mathrm{~mm}$ to $1 \mathrm{~mm}$. It was concluded that the tube vibration and the rotation of the additional rings promote heat transfer. At the same time, the Nusselt number $\mathrm{Nu}$ increases due to the increase of Reynolds number. Lee et.al. [4] performed an practical study to improve the critical heat flux CHF by mechanical vibration of a vertical round tube that was heated under a constant heat flux. The tube was subjected to forced vibration at 0 to $70 \mathrm{~Hz}$ and a vibration amplitude of 0 to $0.001 \mathrm{~mm}$. The study showed that mechanical vibration improves the amount of CHF. This increase depends on vibration amplitude rather than on frequency. Kim et.al. [5] studied the effect of mechanical vibrations with frequencies ranging $5 \mathrm{~Hz}$ to $50 \mathrm{~Hz}$ and a vibration amplitude from $0.05 \mathrm{~mm}$ to $0.5 \mathrm{~mm}$ on critical heat flux $\mathrm{CHF}$ in a vertical annulus tube. It concluded that The vibration of the heat tube leads to an increase in CHF by up to $16.4 \%$. The most effect on CHF is the amplitude of the vibration, where CHF increases with increase of vibration amplitude. Eid and Gomaa [6] carried out a study of the vibration impact was performed to improve the rate of heat transfer from thin planer fins. These fins heated from the base by a heating coil and subject to Vibration ranging from $12.5 \mathrm{~Hz}$ to $50 \mathrm{~Hz}$ and vibration amplitude from $9 \mathrm{~mm}$ to $27 \mathrm{~mm}$. It found that the high frequency vibration increases the percentage of heat transfer rates improvement, while low frequencies have limited effect on improving heat transfer rates. Alaei et.al. [7] performed experimental study to determine the influence of low-frequency vibrations on thermal performance of copper heat pipe subjected to forced vibration at $10 \mathrm{~Hz}$ to $25 \mathrm{~Hz}$ with fixed amplitude at $2 \mathrm{~mm}$. and concluded that the coefficients of heat transfer were increases by boiling and condensation. Sathyabhama and Prashanth [8] experimentally studied the

\footnotetext{
* Corresponding author

This is an open access under a CC BY-NC-SA 4.0 license (https://creativecommons.org/licenses/by-nc-sa/4.0/)
} 
influence of vibration of surface on the coefficient of heat transfer by boiling from a flat horizontal wall for a square boiling chamber with vibration frequency ranging from 0 to 25 $\mathrm{Hz}$ and vibration amplitude from 0 to $4.5 \mathrm{~mm}$. The study reported that increasing of the boiling heat transfer at low frequencies and high amplitudes has reached by $26 \%$. Hosseinian and Isfahani [9] performed practical study to enhancement the heat transfer by surface vibrations at a frequency of $100 \mathrm{~Hz}$ with a vibration level (vibrational acceleration) of $3,6,9 \mathrm{~m} / \mathrm{s}^{2}$ in an elastic dual pipe heat exchanger. It was found that heat transfer coefficient increased by increase the temperature, flow rate, and vibration level and the greatest increase up to $97 \%$ at the highest vibration level in experiment $9 \mathrm{~m} / \mathrm{s}^{2}$. Liu et.al.[10] practically studied the influence of mechanical vibrations on the characteristics of heat transfer of the laminar flow in a copper circular tube exposed to vibration by frequencies ranging from $158 \mathrm{~Hz}$ to $3000 \mathrm{~Hz}$ and accelerations of vibration 1,3 and $5 \mathrm{~g}$. It was found that the influence of frequency on improved heat transfer was larger than the effect of intensity of vibration. The maximum increase in heat transfer $14.94 \%$ is achieved when the Reynolds number is 512 and the vibration frequency is 400 Hz. Hossenian et.al. [11] investigated the effect of the surface vibrations on the stabilization and coefficient of heat transfer of nano-fluid (MWCNTs-water) in an elastic dual pipe heat exchanger. It was concluded that by increasing the flow rate, temperature, concentration of nano-fluid and vibration amplitude, the coefficient of heat transfer increases.

The vibration frequencies from $13 \mathrm{~Hz}$ to $30 \mathrm{~Hz}$ and vibration amplitudes from $0.001 \mathrm{~mm}$ to $0.0022 \mathrm{~mm}$ was used. The most of the literature Related the impact of vibration on the heat transfer process demonstrate that the heat transfer can be enhanced for the laminar internal flow. However, Because of the limited range of frequencies of vibration for which they have been studied, so the frequency and acceleration of the amplitudes are the effectively factors that promote process of heat transfer.

In this paper, a study was carried out for detecting the effect of vertical forced vibrations on the heat transfer by forced convection for laminar internal stream in a spiral fluted tube with constant cross section area and for different levels of heat flux and fluid flow velocity with adoption of purified water as a working fluid. The aims of this study are investigate the followings: (1)The influence of frequency and amplitude of vibration on coefficient of heat transfer, (2)The effect of heat flux and Reynolds number on coefficient of heat transfer, and (3) Based on the experimental data, a new correlation will be obtained

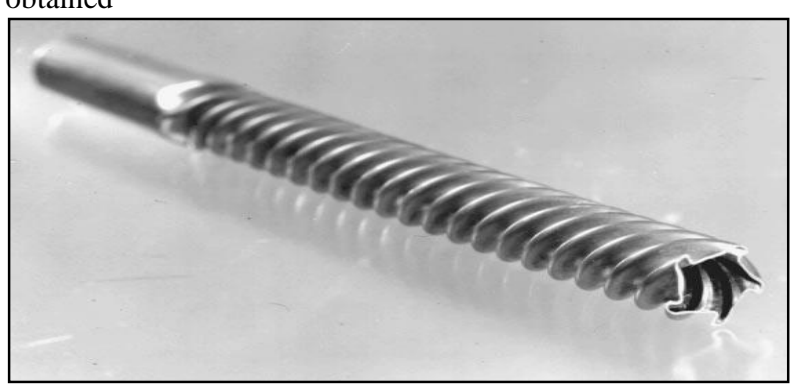

Figure 1. Spiral fluted tube

\section{EXPERIMENTAL APPARATUS}

The system of experimental, As described in Fig.3, consists of a test tube, heating electric circuit, water cycle circuit, temperature measurement, and mechanical vibration generator.

\subsection{Heating electric circuit}

The heated spiral fluted tube, which made from copper was used, with a length of $500 \mathrm{~mm}$, an internal equivalent diameter of $15 \mathrm{~mm}$, an external equivalent diameter of $17 \mathrm{~mm}$, a flute pitch $\mathrm{P}=10 \mathrm{~mm}$, and a flute depth $\mathrm{e}=2 \mathrm{~mm}$, was installed by supported on the vibration generating device. The tube was wrapped with a layer of Furi Adhesive Tape. The layer of insulation was enough thick to ensure that most energy will be supplied transferred immediately to the flowing fluid, then heating wire with a resistance of $45 \Omega / \mathrm{m}$ is wrapped over the layer. The model was completely isolated by a layer of glass wool to reduce the heat loss. A $240-\mathrm{V} \mathrm{DC}$, voltage transformer used to change the power supplied to the heating wire, while maintaining the stability of the voltage entering to the voltage regulator by using voltage stabilizer. Depending on the current, voltage that measured, the heat flux was then determined.

\subsection{Water cycle circuit}

The working fluid in the process of heat transfer was Purified water. flexible pellucid tubes were used to connected the Both ends of the spiral flued tube. The water was withdrawn from the tank by use a centrifugal pump which Can be accurately adjusted using a needle valve. The flowing of Reynolds numbers were used as $228,440,750,1477$, and 1923 respectively. Digital water flowmeter is used to measure the water flow rate. A water tank with a storage capacity of 125 liters was used, the tank supplied with an electric heater to heat the water and reduce the time required to reach the steady state. The temperature inside the tank was maintained at $40^{\circ} \mathrm{C}$, while maintaining the temperature stability by monitoring the temperature inside the tank using Thermometer with 4-Channal.

\subsection{Temperature measurement}

Two thermocouples type-K were accustomed to measure the fluid bulk temperatures by usage drilled punctures at both ends of the tube. By using $18 \mathrm{~K}$-type thermocouples that were installed on the surface of the tube with equal distances using epoxy glue, the mean surface temperature of tube was measured. The temperatures were measured and accumulated using a thermometer with 20 channels, when the system reaches a steady state condition.

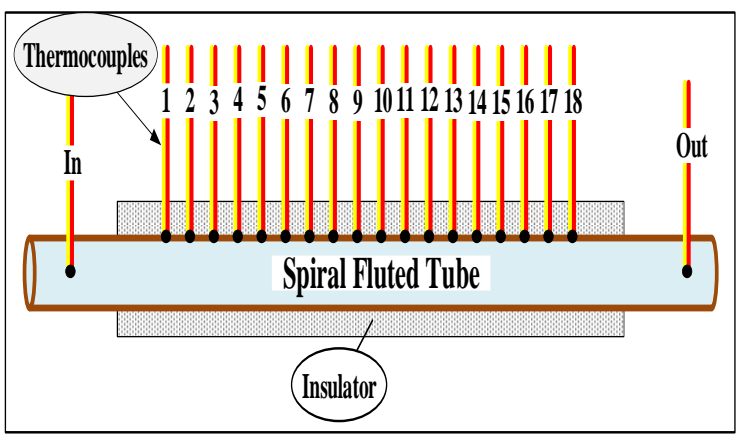

Figure 2. Shows the number and locations of thermocouples on the tube surface.

\subsection{Mechanical vibration generator}

The vibration system is consists of a DC electric motor 24 volt with maximum speed of $4500 \mathrm{rpm}$, a vibration controller which controls the rotational speed of the electric motor and thus controls the vibration frequency applied to the test model, and vibration meter is a Sinometer VM-6360 type, which measures the acceleration of the vibration and thus calculates the vibration amplitude by means of a piezotronic accelerometer that is fixed vertically by magnet tied. In this experiment the parameters of frequency and amplitude were adopted for the range $13 \mathrm{~Hz}<\mathrm{f}$ $<30 \mathrm{~Hz}$ and $0.001 \mathrm{~mm}<\mathrm{Amp}<0.0022 \mathrm{~mm}$, respectively. The period for each test taken more than 15 minutes for purpose reach the steady state condition.

It's worth to mention that the experiments begin at 8:30 am and end at 3:00 pm in each new run when testing a new value of 
frequency to assure that the system has enough time to reach the steady state.

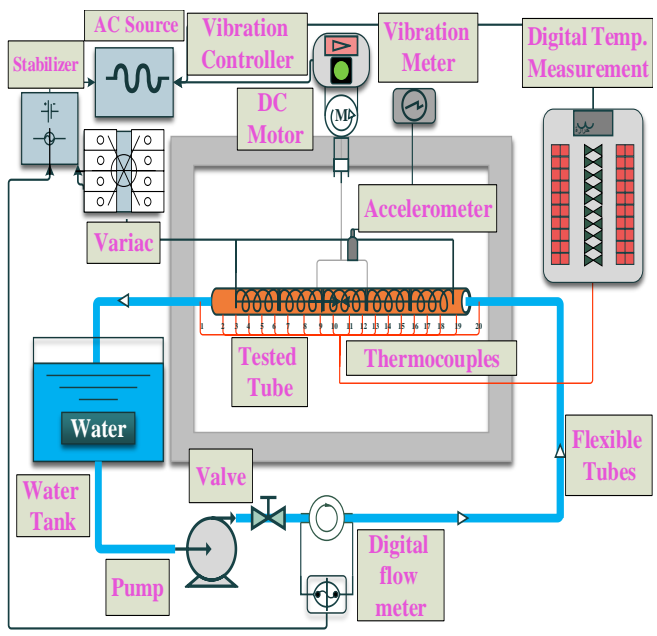

Figure 3. Scheme for testing system

\section{CALCULATIONS OF HEAT TRANSFER}

As illustrated above, the electric power was used to heat the tube, then this heat is transmitted to the tube by conduction and then into the fluid flowing by convection. As the electric power input can be formulated as follows:

$q_{\mathrm{e}}=\mathrm{I} \cdot V_{O}$

where $\mathrm{q}_{\mathrm{e}}, \mathrm{I}$, and $\mathrm{V}_{\mathrm{O}}$ indicates the electrical heat flux, current of electric, and voltage, respectively.

The heat flux applied to the wall is

$q=\frac{q_{\mathrm{e}}}{A_{s, o}}$

Where $A_{s, o}$, is outer surface area of the tube.

The net heat flux of the flowing fluid is obtained by:

$\mathrm{Q}=\rho \cdot u \cdot \frac{\pi D^{2}}{4}\left(T_{0}-T_{i}\right)$

$\rho, u, T_{0}, T_{i}$ are density, velocity, inlet and outlet temperature of the fluid respectively.

The wall tube temperature was calculated as:

$T_{w}=\frac{1}{18}\left(T_{0}+T_{1}+\ldots+T_{18}\right)$

$\mathrm{T}_{1}$ to $\mathrm{T}_{18}$ represents the local temperature of the wall of the tube distributed uniformly in the pivotal orientation of the tube. The average of logarithmic temperatures variation between the tube wall and the bulk temperature is:

$$
\Delta T=\frac{T_{\mathrm{o}}-T_{i}}{\ln \left(\frac{T_{w}-T_{i}}{T_{w}-T_{o}}\right)}
$$

The coefficient of heat transfer for laminar flow is calculated as follows:

$$
h=\frac{Q}{A_{s} \cdot \Delta T}=\frac{\rho \cdot u \cdot \frac{\pi D^{2}}{4} C_{p} \cdot\left(T_{o}-T_{i}\right)}{\pi D \cdot l \cdot \Delta T}
$$

$\mathrm{C}_{\mathrm{p}}$ is a specific heat, $\mathrm{u}$ is a velocity of fluid, $\mathrm{A}_{\mathrm{s}}$ is an inner surface area of the tube.

Because of the complex shape of the inner side of the spiral fluted tube, the concept of "volume based diameters" is used to calculate the equivalent internal diameter, it is calculated as [12]:

$D_{v i}=\sqrt{\frac{4 V}{\pi \cdot l}}$
$\mathrm{V}$ represent the volume enclosed inside fluted tube, and the dimensionless coefficient of heat transfer Nusselt number is:

$N_{u}=\frac{h \cdot D_{v i}}{K}$

Where $\mathrm{k}$ is thermal conductivity of the fluid, and $\mathrm{h}$ is the convective heat transfer coefficient

Reynolds number $(\mathrm{Re})$ can be calculated as:

$\operatorname{Re}=\frac{\rho \cdot u \cdot D_{v i}}{\mu}$

$\mu$ is dynamic viscosity of fluid.

The amplitude of vibration is calculated as:

$\operatorname{amp}_{\bullet}=\frac{a \cdot \sqrt{2}}{(2 \pi \cdot f)^{2}}$

Where a represents the acceleration and $\mathrm{f}$ is the frequency of vibration.

In the equations above, the physical properties of fluid are determined depend on the average fluid temperature and the database property [13].

\section{RESULTS AND DISCUSSION}

\subsection{Validation of experimental results of heat transfer} without vibration.

The heat transfer in laminar flow was studied at the vibration absence, then compared the results of $\mathrm{Nu}$ with the value obtained from the correlation Suggested by 'Sieder and Tate' [14], to check the rigor of our experience before the vibration trial.

$$
N u=1.86\left(\frac{\mathrm{Re} \cdot \operatorname{Pr}}{\frac{l}{D}}\right)^{0.33}\left(\frac{\mu_{f}}{\mu_{w}}\right)^{0.14}
$$

$\operatorname{Pr}$ is prandtl number of the fluid, $\mu_{\mathrm{w}}$ and $\mu_{\mathrm{f}}$ represents the viscosity of the wall fluid and bulk fluid, respectively.

In addition, a correlation was obtained by Churchill and Ozoe [15] for locked stream channel flow comprises jointly the entrance and fully developed zones.

$$
\begin{aligned}
& N u=\left[4.364\left(\frac{G_{z}}{29.6}\right)^{2}\right]^{1 / 6} \\
& *\left[1+\left[\frac{G_{z} / 19.04}{\left[1+\left(P_{r} / 0.0207\right)^{2 / 3}\right]^{0.5}\left[1+\left(G_{z} / 29.6\right)^{2}\right]^{1 / 3}}\right]^{3 / 2}\right]^{1 / 3}
\end{aligned}
$$

Where : $G_{z}=\frac{\pi}{4 x}=\frac{\pi \mathrm{Re} \cdot \operatorname{Pr}}{4 x / D}$

Fig. 4. Shows a comparison between the results of present work with eqns. (11) and (12). The dash line signalizes of our experimental results, whilst the solid lines match to the indicated above correlations. It worthily renowned that the present experience were done in the laminar region, and the flow described as an entrance developing flow. The experimental results were Better reflects with Eq. (11). 


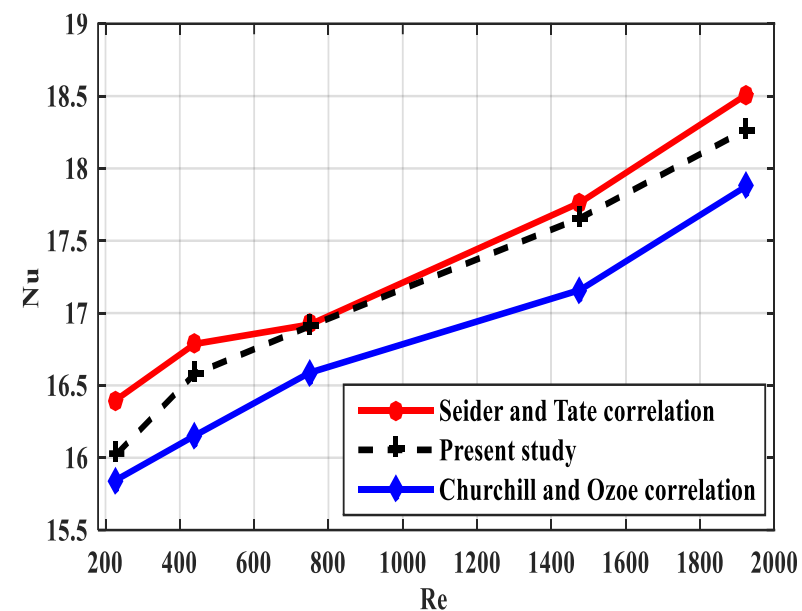

Figure 4. Data Validation of Nusselt number with correlations.

The experiments and correlation meet the criterion of accuracy because the maximum deviation between them is $10.54 \%$.

\subsection{Effect of Reynolds number and heat flux on the non-} dimensional coefficient of heat transfer $(\mathrm{Nu})$.

The effect of the fluid flow represented by Reynolds number and the influence of heat flux on the coefficient of heat transfer was studied. Fig. 5. indicates the influence of Reynolds number on the average rate of heat transfer of the different heat flux adopted in this research. By increasing the Reynolds number, the Nusselt number increases. This is due to the fact that the spiral fluted tube is exposed to maximum flow velocity at the horizontal position. The grooves are paths direct the flow in the horizontal position. When the Reynolds number for the flows is great, the clutter and mixing process of the different layers particles of fluid in the laminar stream are robuster than that at a lower Reynolds number. In addition, the grooves inside the tube act as fins inside the tube and thus generate a swirling motion of the fluid inside the tube and increase the mixing process of the hot fluid at the wall with the cold fluid in the middle of the tube and thus increase the heat transfer process. Also the figure below illustrates the increase of Nusselt number by the heat flux increase due to increasing heat intensity applied on the wall of the tube, where the increasing in Nusselt number was $4.138 \%$ at a heat flux of $618 \mathrm{~W} / \mathrm{m}^{2}, 6.418 \%$ at a heat flux of $1155 \mathrm{~W} / \mathrm{m}^{2}, 10.666 \%$ at a heat flux of $2277 \mathrm{~W} / \mathrm{m}^{2}$, and $13.586 \%$ at a heat flux of $3775 \mathrm{~W} / \mathrm{m}^{2}$.

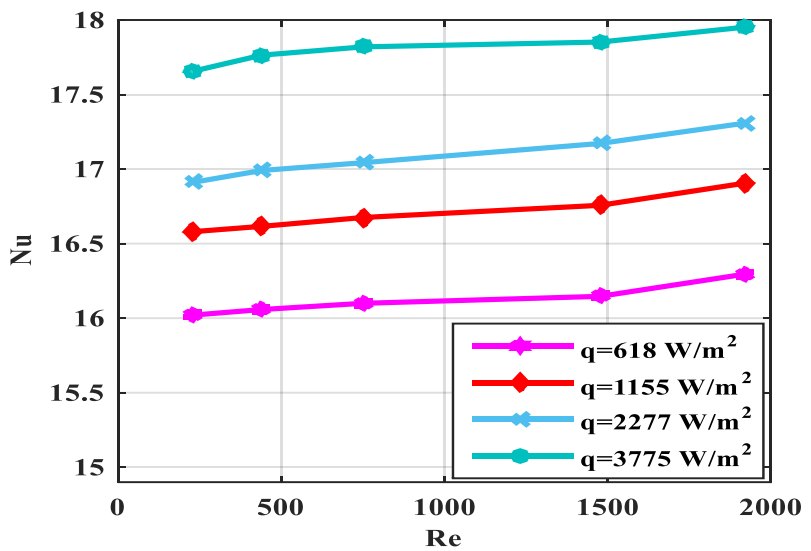

Figure 5. The relation between Nusslet number and Reynolds number without vibration.

4.3 Influence of the vibration frequency and amplitude on the non-dimensional coefficient of heat transfer $(\mathrm{Nu})$.

The impact of forced vibrations on heat transfer coefficient for frequencies $(13,16,20,23,26,30) \mathrm{Hz}$ and different levels of heat flux $\left(618,1155,2277,3775 \mathrm{~W} / \mathrm{m}^{2}\right)$ and Reynolds number $(228,440,750,1477,1923)$ was studied as shown in Fig. 6. It is clear that the increase in the forced frequency increases the non-dimensional coefficient of heat transfer, the coefficient of heat transfer is generally increased when the vibration is exposed than if the sample is not subjected to forced vibration, where the maximum increment occurs at low frequencies and decreases by frequency increases, because the vibration amplitude will be high at low frequency and decreasing as frequency increases. The coefficient of heat transfer decreases by increasing the frequency value, depending on the vibration amplitude. Vibrations with high amplitudes lead to increasing of the heat transfer coefficient, the lower of vibration amplitude means the lower of the coefficient of heat transfer because the low vibrational amplitudes increase the thickness of thermal boundary layer adjacent to the wall and reduces the heat exchange. Thus, the heat transfer coefficient is less. It is clear that the vibrations have an effect on the values of the nondimensional coefficient of heat transfer, Where the percentage

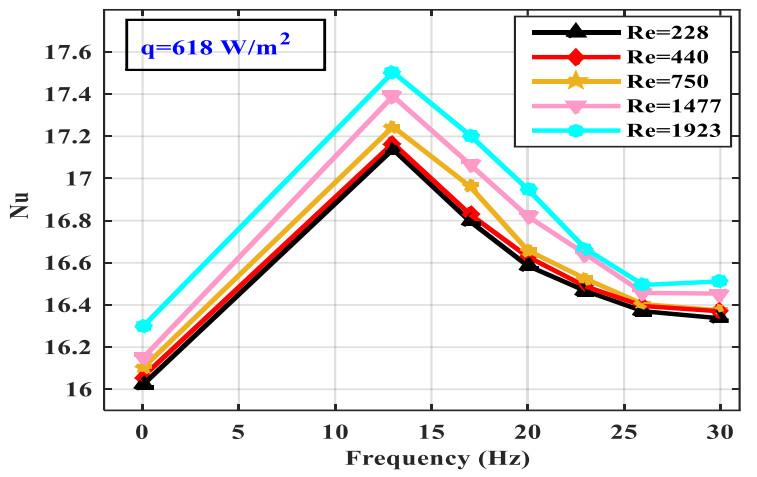

(a)

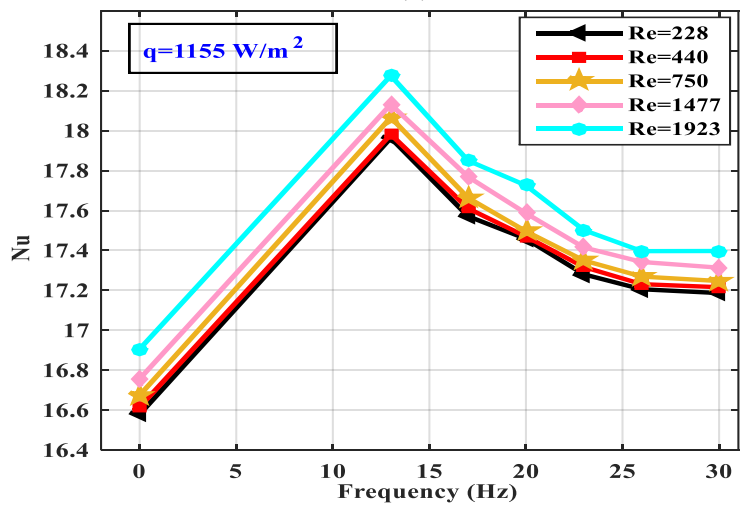

(b)

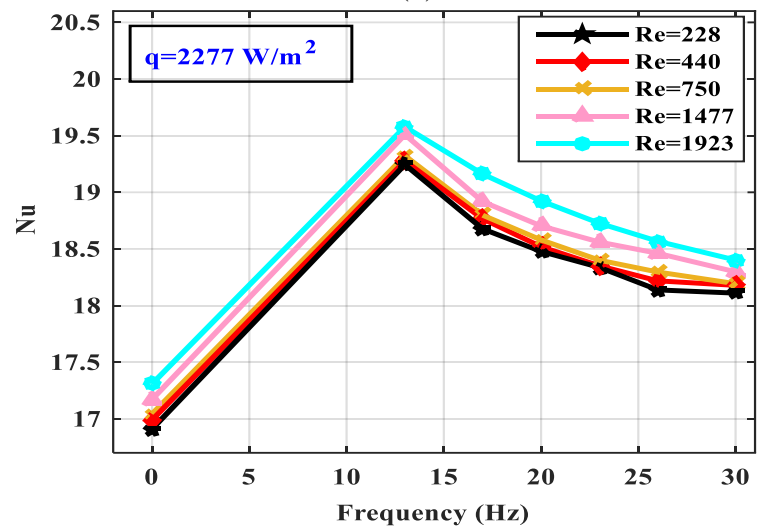

(c) 


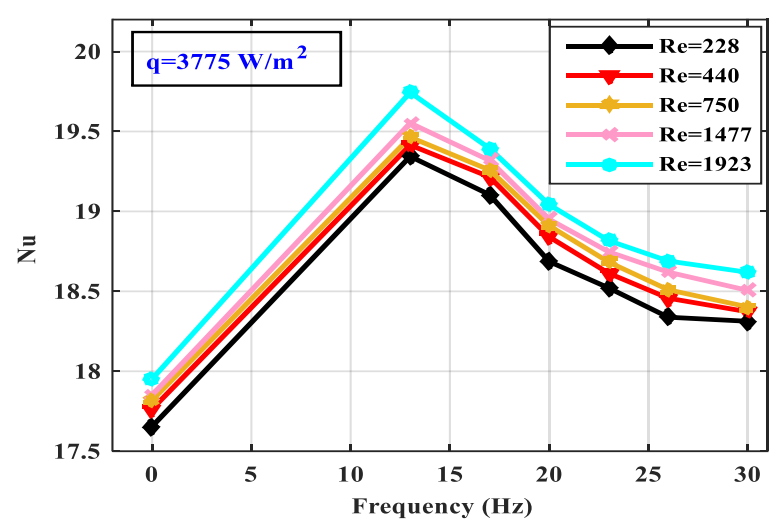

(d)

Figure 6. Relationship among Nusselt number, Reynolds number, heat flux, and frequencies of vibration.

increase in $\mathrm{Nu}$ is $(8.4 \%)$ at $(\mathrm{f}=13 \mathrm{~Hz}),(6.46 \%)$ at $(\mathrm{f}=16 \mathrm{~Hz})$, $(4.71 \%)$ at $(\mathrm{f}=20 \mathrm{~Hz}),(4.09 \%)$ at $(\mathrm{f}=23 \mathrm{~Hz}),(2.48 \%)$ at $(\mathrm{f}=$ $26 \mathrm{~Hz})$ and, $(2.21 \%)$ at $(\mathrm{f}=30 \mathrm{~Hz})$ when a heat flux is 3775 $\mathrm{W} / \mathrm{m}^{2}$, as shown in Fig. 6 . This means that the low frequencies increase the heat transfer coefficient, the large vibration amplitude in the low frequencies leads to breach the thermal boundary layer. Thus, occurrence of the mixing between hot and cold fluid particles, due to vortices by vibration causing an improvement in the heat transfer process. At high frequencies, the vibration amplitude is small and does not breach the thermal boundary layer which represents the impedance of the heat exchange process and leads to heating the tube and the difference in temperatures between the tube surface and the adjacent fluid layer is high, which resulting lower heat transfer. The same behavior seen in the heat flux levels $\left(\mathrm{Q}=1155 \mathrm{~W} / \mathrm{m}^{2}\right.$, $\mathrm{Q}=2277 \mathrm{~W} / \mathrm{m}^{2}$, and $\mathrm{Q}=3775 \mathrm{~W} / \mathrm{m}^{2}$ ). Where the only way to increase the coefficient of heat transfer is by reducing the thermal resistance of the boundary layer and this is done by increasing the disturbance of the main stream so that the turbulence of the vortex enters inside the boundary layer leading to a reduction in total thermal resistance, thus enhancement of the heat transfer process.

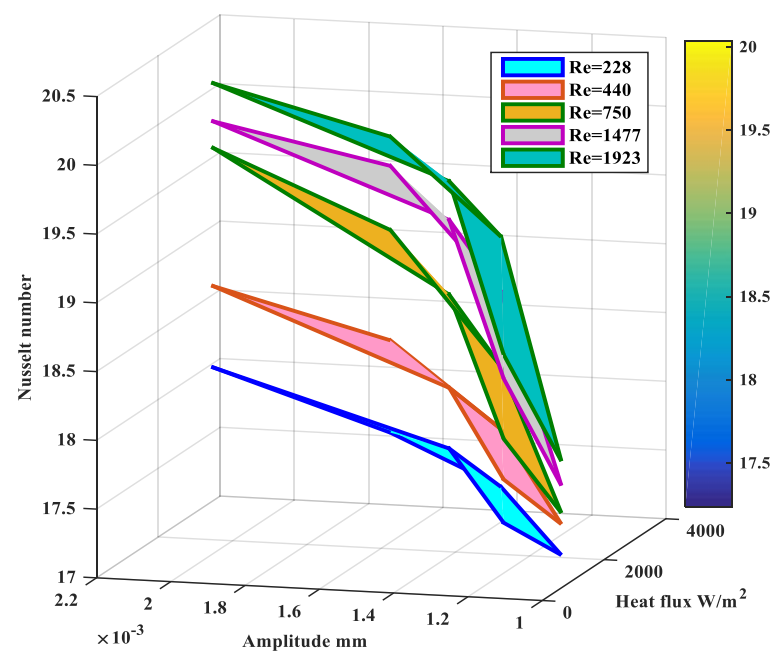

Figure 7. Relationship between Nusselt Number, Vibration amplitude, heat flux, and Reynolds number.

\subsection{System uncertainty analysis}

A root sum square (RSS) method was used to analyze uncertainty for measured quantities. The estimated relative uncertainty of the Reynolds number is found to be $\pm 4.81 \%$, the uncertainty of the non-dimensional heat transfer coefficient $\mathrm{Nu}$ is $\pm 6.26 \%$, and the uncertainty of the heat flux calculated is $\pm 5.17 \%$.

\subsection{Correlation}

Based on the experimental data obtained through 140 tests, a correlation was proposed between the parameters affecting the non-dimensional heat transfer coefficient using the statistical program SPSS, and the value R squared $=1$ - (Residual Sum of Squares) $/$ (Corrected Sum of Squares $)=0.963$. As given by this equation.

$$
N u=12.46297 * \operatorname{Re}^{0.007681 *} q^{0.0696101} * f^{-0.066015}
$$

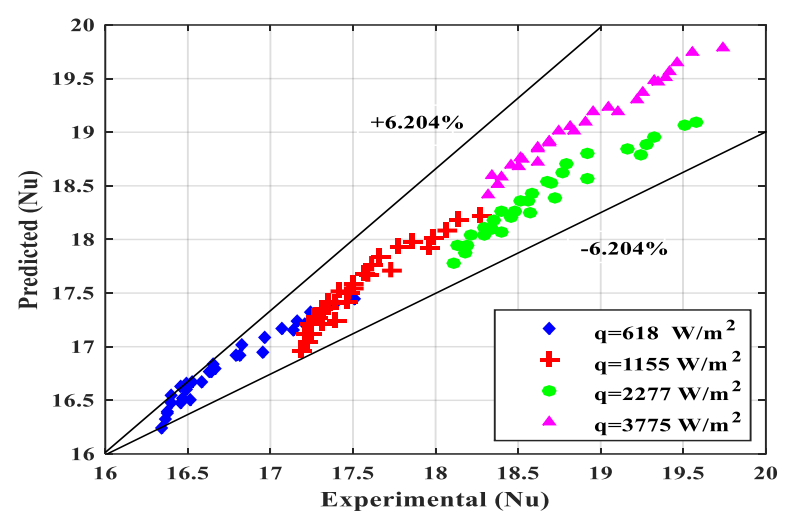

Figure 8. Experimental Nusselt number versus calculated results from correlation.

The comparison with the ref. [10] shows an improvement in the heat transfer of the spiral fluted tube by $52.93 \%$ compared to the smooth tube.

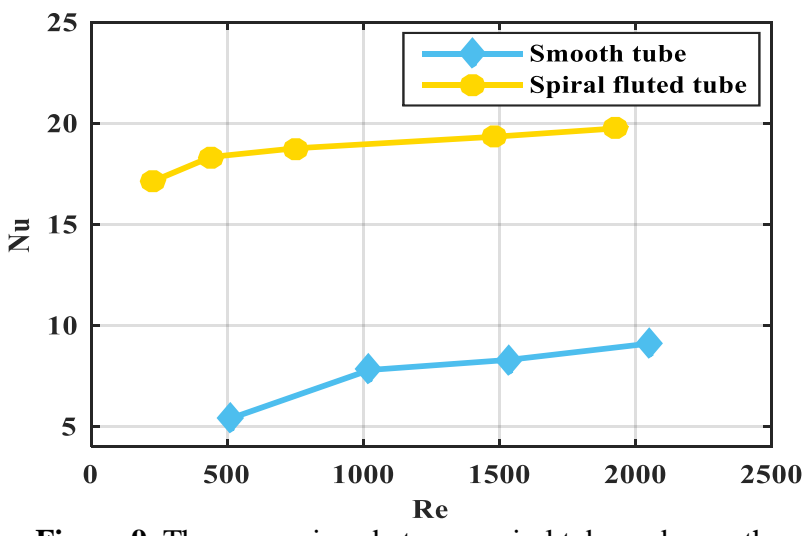

Figure 9. The comparison between spiral tube and smooth tube.

\section{CONCLUSIONS}

The relationship between Nusselt number and Reynolds number of the internal laminar flow of spiral fluted tube was calculated in the absence of vibration. These results were compared with those calculated using two widely recognized correlations and the maximum deviation rate was $10.54 \%$.

The effect of vibration amplitude on heat transfer enhancement proved to be stronger than the vibration frequency effect, due to the ability of the vibration amplitude to penetrate the thermal boundary layer, where reached the highest increase in the $\mathrm{Nu}$ up to $8.4 \%$ at the vibration amplitude $0.0022 \mathrm{~mm}$ and the frequency $13 \mathrm{~Hz}$.

By increasing the heat flux and Reynolds number, $\mathrm{Nu}$ increases, where it reached the highest increase up to $6.02 \%$ at heat flux $3375 \mathrm{~W} / \mathrm{m}^{2}$ and Reynolds number 1923. 
An empirical correlation was established between the independent variables in the research, the average error rate for this equation was $6.024 \%$, which indicated its accuracy.

\section{REFERENCES}

[1] H. J. Kim and J. H. Jeong, "Numerical analysis of experimental observations for heat transfer augmentation by ultrasonic vibration," Heat Transfer Engineering, vol. 27, pp. 14-22, 2006.

[2] W. M. Rohsenow, J. P. Hartnett, and Y. I. Cho, Handbook of heat transfer vol. 3: McGraw-Hill New York, 1998.

[3] L. Bronfenbrener, L. Grinis, and E. Korin, "Experimental study of heat transfer intensification under vibration condition," Chemical Engineering \& Technology: Industrial Chemistry-Plant Equipment-Process Engineering-Biotechnology, vol. 24, pp. 367-371, 2001.

[4] Y. H. Lee, D. H. Kim, and S. H. Chang, "An experimental investigation on the critical heat flux enhancement by mechanical vibration in vertical round tube," Nuclear engineering and design, vol. 229, pp. 47-58, 2004.

[5] D. H. Kim, Y. H. Lee, and S. H. Chang, "Effects of mechanical vibration on critical heat flux in vertical annulus tube," Nuclear Engineering and Design, vol. 237, pp. 982987, 2007.

[6] E. Eid and M. Gomaa, "Influence of vibration in enhancement of heat transfer rates from thin plannar fins," Heat and mass transfer, vol. 45, pp. 713-726, 2009.

[7] A. Alaei, M. H. Kafshgari, and H. Atashi, "A new designed heat pipe: an experimental study of the thermal performance in the presence of low-frequency vibrations," Heat and Mass Transfer, vol. 48, pp. 719-723, 2012.
[8] A. Sathyabhama and S. Prashanth, "Enhancement of boiling heat transfer using surface vibration," Heat TransferAsian Research, vol. 46, pp. 49-60, 2017.

[9] A. Hosseinian and A. M. Isfahani, "Experimental study of heat transfer enhancement due to the surface vibrations in a flexible double pipe heat exchanger," Heat and Mass Transfer, vol. 54, pp. 1113-1120, 2018.

[10] W. Liu, Z. Yang, B. Zhang, and P. Lv, "Experimental study on the effects of mechanical vibration on the heat transfer characteristics of tubular laminar flow," International Journal of Heat and Mass Transfer, vol. 115, pp. 169-179, 2017.

[11] A. Hosseinian, A. M. Isfahani, and E. Shirani, "Experimental investigation of surface vibration effects on increasing the stability and heat transfer coeffcient of MWCNTs-water nanofluid in a flexible double pipe heat exchanger," Experimental Thermal and Fluid Science, vol. 90, pp. 275-285, 2018

[12] P. Rousseau, M. Van Eldik, and G. Greyvenstein, "Detailed simulation of fluted tube water heating condensers," International Journal of Refrigeration, vol. 26, pp. 232239, 2003.

[13] E. W. Lemmon, M. L. Huber, and M. O. McLinden, "NIST reference fluid thermodynamic and transport propertiesREFPROP," NIST standard reference database, vol. 23, $\mathrm{p}$. v7, 2002.

[14] E. N. Sieder and G. E. Tate, "Heat transfer and pressure drop of liquids in tubes," Industrial \& Engineering Chemistry, vol. 28, pp. 1429-1435, 1936.

[15] A. Bejan, Convection heat transfer: John wiley \& sons, 2013 